\title{
La Naturaleza puesta en Jaque: La Expansión de la Frontera Agropecuaria en Argentina y su Impacto Socio-Ambiental (1980- 2017)
}

Adrián Zarrilli ${ }^{1}$

\section{RESUMEN}

La expansión de la frontera agrícola asociada con el notable crecimiento de los productos agrícolas en Argentina a finales de los siglos XX y XXI, impulsada principalmente por la gran expansión del cultivo de soja, ha producido uno de los mayores cambios económicos, sociales, demográficos y ambientales en la Argentina contemporánea. La soja, como cultivo dominante de las últimas décadas en la agricultura argentina, ha sido ponderada por algunos sectores y demonizada por otros. Para algunos, es el cultivo que ha permitido la recuperación de la economía argentina debido a su alta productividad, rentabilidad y su impacto positivo en la balanza comercial de las últimas dos décadas. Destacan la flexibilidad del cultivo, la simplicidad de su manejo y el alto impacto de su paquete tecnológico, sostenido en un modelo integrado por siembra directa, semillas transgénicas y la aplicación generalizada de herbicidas. Para otros en cambio, el cultivo de esta oleaginosa desencadena la deforestación rápida de los bosques nativos, concentra la riqueza en grandes corporaciones, destruye empleos rurales, expulsa a las comunidades indígenas, aniquila la diversidad biológica, deteriora la calidad ambiental y afecta la salud humana. ¿Cuánto hay de mito y realidad en este debate a menudo sesgado por intereses económicos, políticos o ideológicos? Este trabajo busca aclarar algunos aspectos críticos y controvertidos de este debate. Es clave tratar de responder algunas preguntas sustanciales: ¿Cuánto de apología y realidad hay en los impactos ambientales de este modelo sojero, en relación con la biodiversidad, el uso de pesticidas y los cambios en el hábitat? ¿Qué transiciones sociales produce en el espacio rural argentino? ¿Cómo se cotejan los indicadores ecológicos y ambientales con otros países del subcontinente y del mundo? Ninguno de estos problemas tiene una respuesta simple, pero es posible tratar de darle forma a través del análisis de los 30 años de progreso de la frontera agrícola.

Palabras clave: Argentina; Región Chaqueña; Soja; Ambiente. 
"El pueblo que confía su subsistencia a un solo producto, se suicida" José Martí

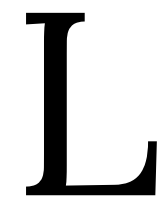

a expansión de la frontera agrícola asociada al importante crecimiento de los productos agrícolas en Argentina a fines del siglo XX y XXI, impulsada principalmente por la gran expansión del cultivo de soja, ha producido uno de los mayores cambios económicos, sociales, demográficos y ambientales en la Argentina contemporánea.

¿Bendición o maldición? La soja, como cultivo dominante de las últimas décadas en la agricultura argentina, ha sido celebrada por algunos sectores, como demonizada por otros. Para algunos, es el cultivo que ha permitido la recuperación de la economía argentina debido a su alta productividad y rentabilidad y a su impacto positivo sobre los excedentes generados en la balanza comercial de las dos últimas décadas. Destacan la flexibilidad del cultivo, la sencillez de su manejo y el alto impacto de su paquete tecnológico, sostenido en un modelo integrado por la siembra directa, el uso de material transgénico resistente, y la aplicación generalizada de herbicidas. Para otros, el cultivo de esta oleaginosa dispara una rápida deforestación de los bosques nativos en el norte del país, concentra la riqueza en grandes corporaciones, destruye empleos rurales, expulsa comunidades indígenas, aniquila la diversidad biológica, y deteriora la calidad ambiental debido a un mayor uso de plaguicidas y fertilizantes que contaminan el suelo, el aire y los cuerpos de agua, y afectan la salud humana ¿Cuánto hay de mito y realidad en esta puja a menudo sesgada por intereses económicos, políticos o ideológicos?

Este trabajo procurará esclarecer algunos aspectos críticos y controversiales de este debate. Resulta clave tratar de responder, a través de la rigurosidad del conocimiento científico disponible, algunas cuestiones críticos: ¿Cuánto hay de mito y de realidad en los impactos ambientales de este modelo sojero? ¿Aumenta la contaminación por plaguicidas? ¿Aumenta la erosión de los suelos por el mayor cultivo? ¿Se incrementa el procesos de deforestación en Argentina? ¿Se pierden áreas de pastizales y pasturas? ¿Cuán afectados están el hábitat y la biodiversidad? ¿Hay una pérdida excesiva de minerales esenciales como el carbono, el nitrógeno y el fósforo? ¿Cuál es el impacto real del cultivo de soja sobre el ambiente y las poblaciones 
rurales? ¿Cómo están los indicadores ecológicos y ambientales respecto a otros países del subcontinente y del mundo? Ninguna de estas cuestiones tiene una respuesta simple, pero sí es posible intentar conformarla a través del análisis de 50 años de avance de la frontera agropecuaria

\section{El Avance Del Monocultivo de Soja en el Cono Sudamericano}

Debido a la alta rentabilidad de la soja y gracias a las tecnologías que se han desarrollado a su alrededor, este cultivo se extendió a áreas donde la agricultura no se creía posible. En muchas de estas áreas, la expansión de la soya se lleva a cabo a expensas del reemplazo de los ecosistemas nativos, como los bosques, praderas y humedales, así como el reemplazo de otras actividades productivas que son menos rentables. Este proceso genera un mayor uso de agroquímicos y amenaza a las comunidades que dependen de estos ecosistemas para su sustento.

Desde principios de los años 80, el cultivo de soja en América del Sur aumentó exponencialmente en aproximadamente un 300\%. Este aumento fue el resultado de la expansión de la frontera agrícola y el reemplazo de otras actividades productivas en áreas ya transformadas en Argentina, Bolivia, Brasil y Paraguay. En estos cuatro países, el área plantada con soja constituye actualmente más del 50\% del área total plantada con los cultivos más importantes, un porcentaje que ha aumentado significativamente en la última década. Para el ciclo 2017/18, estos cuatro países superaron los 60 millones de hectáreas cultivadas, siendo Brasil y Argentina los países con la tasa de crecimiento promedio anual más alta.

La crisis ambiental en la que nos encontramos es consecuencia de los reclamos de la dominación técnica del planeta vivo que habitamos, que se remontan a la ruptura fundamental que el pensamiento occidental se comprometió a desprenderse de la inmanencia del espacio terrestre, y que en la era moderna se traduce en el poder ilimitado de cálculo, planificación, control, manipulación y dominación de la naturaleza. La revolución verde, iniciada en la década de 1960, es un excelente ejemplo de esta forma particular en que la cultura hegemónica se relaciona con el orden del ecosistema. Esta revolución se refiere a un paquete de tecnologías 
que incluía la introducción de variedades vegetales de alto rendimiento, riego o suministro controlado de agua, el uso de fertilizantes químicos y pesticidas, el uso extensivo de maquinaria agrícola, así como la "mejora" de la genética, nutrición y sanidad animal. El intento del agronegocio de someter la naturaleza y modificarla siguiendo los mandatos de acumulación de capital para maximizar los rendimientos ha demostrado que la aparente eficacia y utilidad de la tecnología de la revolución verde ha terminado siendo interrumpida en su antítesis: la devastación de la Tierra, la desertificación de las fuerzas vitales y el colapso progresivo de las condiciones ecológicas que debemos mantener, que consiste en que la aspiración moderna de dominar la naturaleza no ha hecho más que producir desecación y roer los marcos vitales ${ }^{2}$.

La actividad agro-biotecnológica es una fuerza que ordena a las plantas, animales y personas que constituyan un conjunto regulado y disciplinado de seres vivos que operan por el bien de un modelo que impone el patrón de la fábrica a la naturaleza ${ }^{3}$. Ejerce poder sobre la vida, ahora no solo desplazando especies durante siglos en nichos ecológicos sino también manipulando y modificando el código genético. La ciencia de la biotecnología al servicio del capital corporativo interviene y transforma los genes para que algunas transnacionales puedan ejercer poder sobre la esencia de la vida, apropiándose, como si ahora fueran su autoría ${ }^{4}$.

En el caso de Argentina, el proceso de expansión de la frontera agrícola ha sido un movimiento dinámico, condicionado por condiciones de mercado favorables y por la disponibilidad de tierras adecuadas y baratas. Desde finales de la década de 1990, Argentina casi triplicó el volumen de sus cultivos de varios granos, mientras que al mismo tiempo aumentaron significativamente los efectos sociales adversos como la pobreza y la indigencia, entre otros problemas.

En este contexto de intensa transformación, la región pampeana continuará siendo el principal centro productivo del país. Sin embargo, los cambios están afectando a otras áreas, y dentro de ellas, especialmente a la región del Chaco, donde

\footnotetext{
2 Omar Felipe Giraldo, Ecología Política de La Agricultura. Agroecología y Posdesarrollo (San Cristóbal de Las Casas, Chiapas, México: El Colegio de la Frontera Sur, 2018).

${ }^{3}$ Vandana Shiva, Los Monocultivos de La Mente (Monterrey, Mexico: Editorial Fineo, 2007).

${ }^{4}$ Giraldo, Ecología Política de La Agricultura. Agroecología y Posdesarrollo.
} 
es posible anticipar un aumento notable en la actividad agrícola y ganadera, tanto por el potencial productivo de sus tierras, como por la diferencia en los ingresos, resultante del precio de los campos, mucho más bajos que los de la región pampeana. Como consecuencia, los procesos que generan transformaciones notables y cuestionables están ocurriendo en la región citada:

- en el paisaje natural donde se multiplican las áreas desmontadas, producto del avance significativo de la frontera agrícola;

- en el paisaje agrario, debido a la fuerte caída en el área sembrada con algodón y el marcado predominio de la soja;

- en el modelo de ocupación del territorio, donde en lugar de las antiguas colonias de pequeños productores, predominan los establecimientos grandes y medianos;

- en la fuerte caída de la población rural (porque la soja ocupa menos mano de obra que el algodón), lo que genera migraciones a los cinturones de pobreza de las grandes ciudades

\section{El Proceso de la Agricultura y la ExPANSión de la Frontera Agrícola}

La actividad agrícola pampeana desempeñó un papel central en el suministro de alimentos al mercado nacional e internacional y al desarrollo general de Argentina. La pampa húmeda es una llanura de más de 60 millones de hectáreas (casi el 22\% del territorio continental argentino), cuya riqueza y potencial productivo le dieron ventajas comparativas sustanciales para la producción agrícola. Cuatro actividades dominan las tierras pampeanas: agrícola (cereales y oleaginosas), ganadería (cría, invernada y lácteos), urbana y periurbana.

El sector agrícola, agroalimentario y agroindustrial argentino (SAAA) tiene una relevancia estratégica para el país. Durante la campaña 2014/15, el sector agrario superó con creces los 100 millones de toneladas de granos (el 53\% corresponde a semillas oleaginosas, el resto a cereales y otros granos). Debido a sus condiciones naturales favorables, su historia en la producción agrícola y su capital humano en el sector agrícola y agroindustrial, Argentina se posiciona como uno de los líderes en los 
mercados internacionales de productos agroalimentarios. La importancia estratégica de la agricultura en la economía argentina es evidente teniendo en cuenta que el sector aporta más del $50 \%$ del valor total de las exportaciones y tiene una participación aproximada del 7\% sobre el Producto Interno Bruto (PIB) total, que llegaría a 18-22 \% si se agrega la contribución neta indirecta agregando la cadena de agroprocesamiento. Al mismo tiempo, emplea directamente a aproximadamente el 7\% de la fuerza laboral registrada, a lo que se debe agregar el empleo asociado con las actividades agroindustriales, lo que elevaría este porcentaje al $17 \% 5$.

Hasta la década de 1930, la expansión de la agricultura argentina se centró en el desarrollo de su frontera agrícola mediante la ocupación de nuevas tierras, con un $30 \%$ del total de la tierra agrícola-ganadera en las pampas húmedas. Después de un período de estancamiento agrario de casi tres décadas a partir de los años 60, la expansión agrícola se produjo a expensas del área dedicada a la ganadería extensiva y ha ocupado en los últimos años más del $50 \%$ del espacio productivo de la pampa húmeda ${ }^{6}$. Esta sustitución causó el desmantelamiento de una proporción significativa de la infraestructura de reproducción bovina en la zona del núcleo Maicero (casi 5 millones de hectáreas con alta fertilidad que fueron el epicentro del desarrollo de la agricultura en el período moderno). La agricultura se consolidó por primera vez en las pampas húmedas, siendo en los años 70 cuando el proceso comenzó a implementarse en otras regiones ecológicas del país. Allí, se trasladaron capital, tecnologías de producción, parte de la producción de carne de res y granos y plántulas de forraje?

En la década de 1990, el proceso de concentración de la tierra se aceleró y la actividad agrícola se intensificó, tanto en las pampas húmedas como en las regiones extra-pampeanas (noroeste y noreste). Los censos agrícolas del Instituto Nacional de Estadística y Censos (INDEC) muestran que entre 1988 y 2002 el área promedio de fincas aumentó de 375 a 509 hectáreas en las provincias pampeanas ${ }^{8}$. Esta

\footnotetext{
5 Julio J. Nogués, "Barreras Sobre Las Exportaciones Agropecuarias: Impactos Económicos y Sociales de Su Eliminación" (Argentina, 2015), $\mathrm{http} / / /$ documentos.bancomundial.org/curated/es/821761468000270036/Barreras-sobre-las-exportaciones-agropecuarias-impactos-económicos-ysociales-de-su-eliminación.

6 Jorge E. Rabinovich and Filemón Torres, "Caracterización de Los Síndromes de Sostenibilidad Del Desarrollo. El Caso de Argentina, Santiago, ECLAC, the Case of Buenos Aires," Environment and Urbanization 12, no. 2 (2004): 43.

7 J. Morello, "Entrando Al Chaco Con y Sin El Consentimiento de La Naturaleza," Vida Silvestre 92 (2005): 23.

${ }^{8}$ Martín Piñeiro and Feredico Villarreal, "Modernización Agrícola y Nuevos Actores Sociales," Revista Ciencia Hoy En Línea 15, no. 87 (2005): $32-$ 36.
} 
concentración productiva ha sido acompañada por una tremenda transformación agrícola y el aumento sostenido en el cultivo de soja. En los últimos años, casi 7 millones de hectáreas de campos de cultivos naturales o forrajeros, utilizados para la producción de carne (casi el 15\% del ganado total) se han convertido a la agricultura solo en la pampa húmeda. La expansión de la soya ha llevado a una caída en la siembra de maíz entre 10 y 20\% porque los costos de producción del maíz son el doble que los de la soja.

La producción total del país casi se cuadruplicó en poco más de 30 años, lo que implica un crecimiento de aproximadamente $2.5 \%$ anual. El aumento de la productividad y el cambio tecnológico han jugado un papel importante en el crecimiento de la agricultura argentina. El aumento de la producción agrícola se ha mantenido y promedió una tasa de crecimiento anual acumulada del 3\% entre 1962 y 2013. Este impulso de la producción a largo plazo se explica, por un lado, por el aumento en el uso de factores e insumos (tierra, mano de obra, capital, fertilizantes, etc.), cuya tasa de crecimiento ha sido del $0,62 \%$ anual y, por otro lado, mucho más crítica, debido al aumento de la productividad total que aumentó un 2,5\% anual . Es decir, una buena parte del aumento de la producción agrícola puede explicarse por mejoras en la productividad, lo que implica una mayor capacidad de producción del conjunto de recursos disponibles ${ }^{9}$.

En este contexto, existe el llamado proceso de "agriculturización", que se define como el uso creciente y continuo de la tierra para cultivos en lugar de ganado o propósitos mixtos. También se asocia en las pampas con los cambios tecnológicos, la intensificación del ganado, la expansión de la frontera agrícola hacia las regiones extra-pampeanas y fuertemente relacionadas con la sostenibilidad, la tendencia a las producciones orientadas al monocultivo, principalmente soja o la combinación trigosoja.

\footnotetext{
${ }^{9}$ Daniel Lema, “Crecimiento y Productividad Total de Factores En La Agricultura: Argentina y Países Del Cono Sur 1961-2013" (Argentina, 2015), http://documents.worldbank.org/curated/pt/970151468197997810/Crecimiento-y-productividad-total-de-factores-en-la-agricultura-Argentina-ypaíses-del-cono-sur-1961-2013.
} 
Mapa 1. Areas de producción de soja en Argentina, 2017.

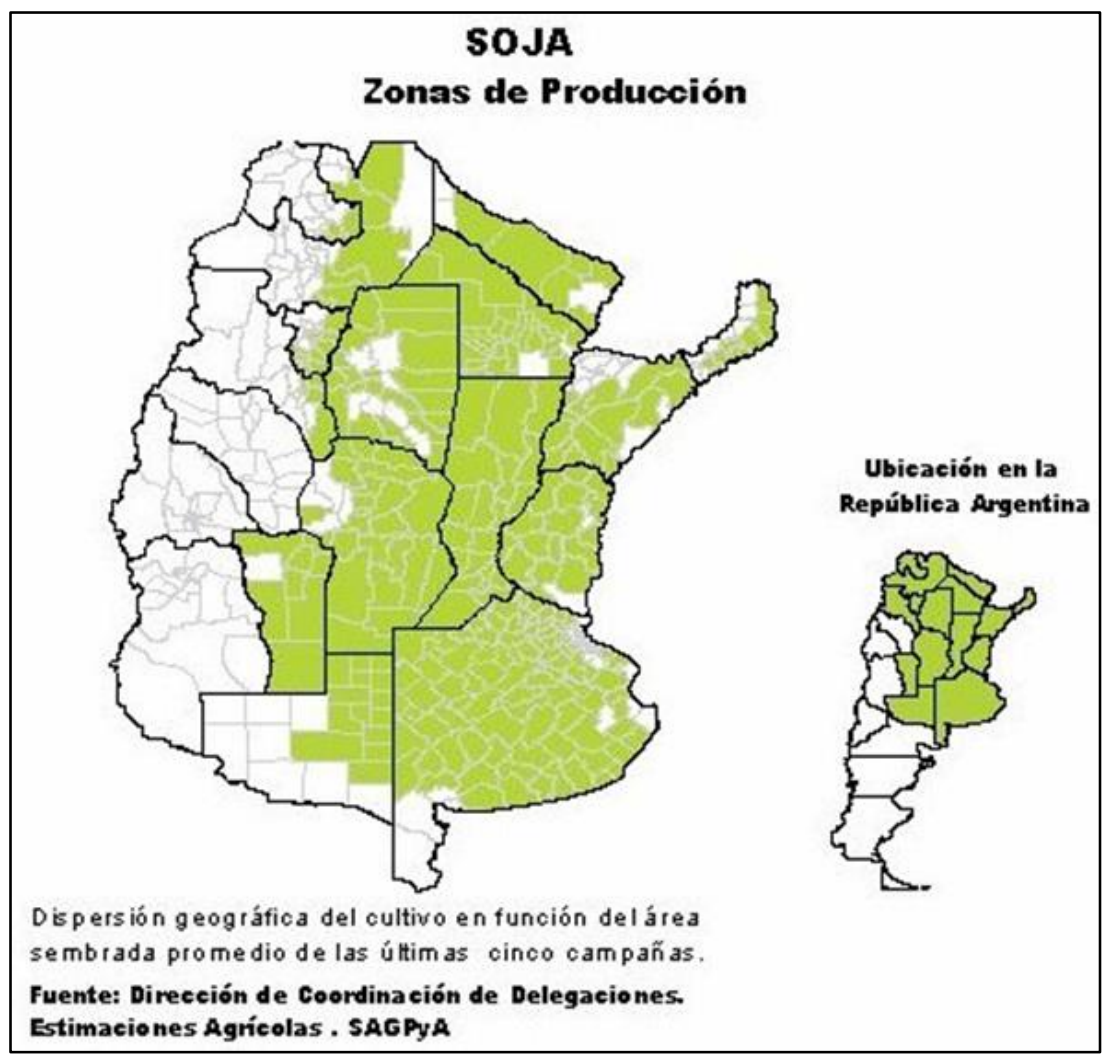

Fuente: Dirección de Coordinación de Delegaciones. Ministerio de Agricultura, Ganderia, Pesca y Alimentación, Buenos Aires (2017).

Uno de los cambios cualitativos sustanciales en este contexto es que el eje de la explotación agrícola no se centra en la propiedad de la tierra, sino en la capacidad del productor-empresario para organizar y coordinar una red de contratos. Incluso en los casos de productores que poseen la tierra que trabajan, la estrategia económica y financiera habitual es la de un organizador de contratos o "grupo de siembra", vinculado a varios mercados: capital para la financiación de la tierra para el arrendamiento y servicios en los que los contratistas son oferentes. Esta estrategia ha favorecido la combinación de sistemas de tenencia de la tierra que tienden a aumentar el área trabajada sin necesariamente expandir la escala de propiedad. En la región central, los contratistas adquirieron gran relevancia a partir de finales de la década de 1980, a medida que la demanda de trabajo agrícola para el que el propietario no posee maquinaria se expandió.

No es posible considerar las transformaciones ocurridas en el agro argentino desde las últimas décadas del siglo pasado sin hacer referencia a las grandes diferencias entre las regiones agroecológicas del país, porque la modernización 
tecnológica y la transformación del mercado interno tuvieron efectos diferentes en las diversas regiones y entre productores y trabajadores. La región pampeana (comprendiendo las provincias de Buenos Aires, Córdoba, Santa Fe y La Pampa) es la que ha provisto la mayor parte de la producción agraria en cuanto a granos y productos ganaderos, y la casi totalidad de las exportaciones agropecuarias (primarias y manufacturadas). Ha resultado particularmente favorecida - aunque con fuertes heterogeneidades internas- por las alzas registradas en oleaginosos y algunos cereales, mientras el decrecimiento de los cultivos industriales que se destinaban principalmente al mercado interno (algodón, caña de azúcar o yerba mate, entre otros) afectó las economías regionales no pampeanas, es decir, al resto de las regiones: el Noroeste, el Noreste, Cuyo y la Patagonia. Ello se debió a la creciente importancia del complejo sojero y al incremento de la industria aceitera para la exportación, situación que no ha sido seguida con igual resultado por las producciones regionales en las que se observó un relativo estancamiento, con pocas excepciones.

Si bien el área sembrada con soja tiene una amplia dispersión, es en la región del Parque Chaqueño donde se registra su explosiva expansión. Motiva lo anterior, además de los avances biotecnológicos que posibilitan expandir el cultivo sobre nuevas áreas, el marcado diferencial en cuanto al valor de la tierra. Mientras que en la zona núcleo (Sur de Córdoba y Santa Fe y Norte de Buenos Aires) los campos oscilan entre los U\$S 6.000 y U\$S 18.000/ha; en Salta, Chaco o Santiago del Estero su precio varía entre U\$S 200 y U\$S $1.500 / \mathrm{ha}^{10}$. No resulta entonces casual que el área sembrada en la Pampa Húmeda haya aumentado un 85\% entre las campañas 1997/98 y $2004 / 05$, mientras que en el noroeste lo haya hecho un $220 \%$, en el noreste un $417 \%$ y en otras regiones marginales un $522 \%{ }^{11}$. Este "complejo sojero" tiene como elementos constitutivos la utilización de semillas mejoradas, agroquímicos y maquinaria de alta capacidad operativa, y la continua adopción de los cultivos transgénicos. Más de 150.000 pequeños y medianos productores han desaparecido en

\footnotetext{
${ }^{10}$ Celina Montenegro et al., "Estimación de La Pérdida de Superficie de Bosque Nativo y Tasa de Deforestación En El Norte de Argentina" (Buenos Aires, 2005).

11 Carlos Merenson, "Primera Estimación Del Pasivo Socio-Ambiental de La Expansióndel Monocultivo de Soja En Argentina," Ciencia \& Naturaleza 11 (2009): 1-7.
} 
poco más de una década al no poder "adaptarse" a esta situación macroeconómica con altos impuestos, elevados precios de los insumos y dependencia de precios internacionales, todas ellas variables fuera de su control. Cerca de 400.000 personas que dependían de la agricultura, no sólo para obtener alimento sino para mantener viva su identidad cultural, han migrado a las grandes ciudades o se mantienen en la pobreza en sus propios predios.

Gráfico 1. Producción de soja en la República Argentina, 1969-2015.

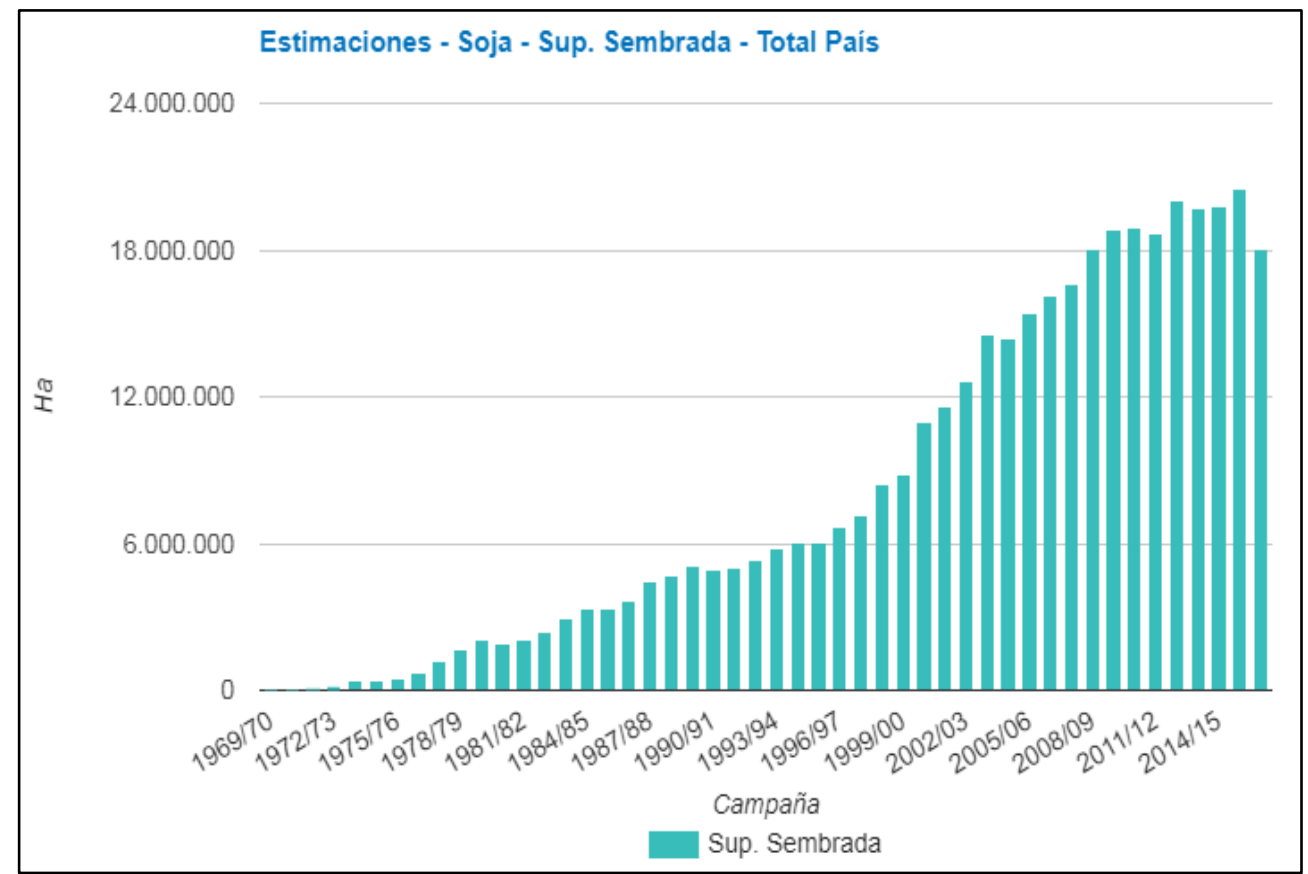

Fuente: Elaboración propia en base a Ministerio de Agricultura, Ganadería, Pesca y Alimentación, Buenos Aires (2016).

En muchos casos la caída de la rentabilidad y el endeudamiento determinaron la cesión de los predios a actores económicos nuevos en la actividad agrícola: fondos de inversión nacionales y extranjeros, pools de siembra, grandes empresas transnacionales que vieron en la "agricultura industrial" argentina un espacio económico en el cual era posible realizar negocias rentables, seguros y a corto plazo. Dado que en estos actores económicos prima la rentabilidad económica de corto plazo, y dado el impacto de sus prácticas sobre los recursos naturales, se exacerba el desarrollo de una agricultura de tipo extractivo en la Argentina. Se desarrolla entonces un sistema de "agricultura sin agricultores"12, donde se sobrepone la

\footnotetext{
12 Walter A. Pengue, "Commoditización y Diversificación de La Producción Agropecuaria Frente a La Oferta Biotecnológica Argentina: Dos Alternativas Mutuamente Excluyentes?," in Transgénicos. Biotecnología En El Agro, ed. Comisión de Agricultura de la Honorable Camara de Senadores de Buenos Aires (Buenos Aires: Editorial Universitaria de La Plata, 2000), 23.
} 
rentabilidad cortoplacista y el uso irracional de los recursos al uso sustentable. Una de sus principales consecuencias ha sido la concentración de tierras en un cada vez menor número de empresas y la importancia creciente del capital extranjero. La concentración económica también ha llevado a que grandes monopolios integrados verticalmente (provisión de insumos, producción, distribución y procesamiento) dominen la escena productiva, relegando a los productores a la ejecución de etapas menos rentables o más riesgosas.

Asimismo durante el primer "boom de la soja" de los años 80, los pastizales en las provincias de la Pampa fueron transformados a la agricultura arable o de labranza. El arado ocasionó una erosión y degradación generalizadas de la tierra, con los consecuentes impactos adversos, río abajo, de sedimentación e inundaciones. Para remediar este problema se introdujeron técnicas de siembra directa o de labranza cero, pero el control de malezas resultó difícil, especialmente en un sistema donde se siembran continuamente cultivos anuales. Cuando en 1998 se introdujo la soja OGM o genéticamente modificada, tolerante a los herbicidas, ésta fue rápidamente adoptada por los agricultores argentinos ${ }^{13}$. La resistencia de la soja OGM a los glifosatos facilitó el control de malezas, de modo que para 2002 la adopción de la soja OGM llegó a acercarse al 100\%.

Además de la pérdida de los hábitats naturales, el explosivo crecimiento del cultivo de soja en Argentina ha tenido otras consecuencias socioeconómicas severas. La producción de alimentos y de lácteos para el mercado nacional se desplomó, en tanto que se incrementó el uso de los agroquímicos, la intoxicación humana y la contaminación del agua. La combinación de crisis económica y expulsión de los pequeños agricultores y de los trabajadores rurales, resultante de la siembra mecanizada de soja, ha disminuido la soberanía alimentaria e incrementando la pobreza y el hambre ${ }^{14}$.

La diferencias ambientales y sociales entre la pampa húmeda y las regiones extra-pampeanas, al norte del país, hacen que el modelo de expansión agrícola

\footnotetext{
${ }^{13}$ Sue Branford, "Argentina's Bitter Harvest," NewScientist, 2004, 34, https://www.newscientist.com/article/mg18224436-100-argentinas-bitterharvest/.

14 Jan Maarten Dros, Manejo Del Boom de La Soya: Dos Escenarios Sobre La Expansión de La Producción de La Soya En América Del Sur (Amsterdam: AIDEnvironment, 2004), 23.
} 
presente particularidades para éstas últimas, las que deben destacarse explícitamente cuando se habla de "pampeanización", para referirse a la agriculturización basada en la exportación indiscriminada del modelo de producción pampeano a regiones extrapampeanas. Los principales efectos de la agricultura argentina sobre el ambiente incluyen la degradación de los suelos, la contaminación con agroquímicos, la deforestación, la pérdida de biodiversidad, las emisiones de gases de efecto invernadero y los problemas derivados del uso de agua dulce ${ }^{15}$.

Los fenómenos de agriculturización y pampeanización presentan una estructura causal muy similar. En general, estos diferentes significados o intensidades apuntan a que las amenazas y tendencias de insosteniblidad en el caso de las regiones extra-pampeanas son mucho más marcadas.

En primer lugar, en el caso de las regiones del norte, los precios relativos de las tierras son mucho menores que en la pampa. Esta trasformación genera que el progreso de la superficie sembrada se dé con gran rapidez. Además, este impulso acelerado no sólo se produce a costa de otros cultivos sino también a través del desmonte y la eliminación de fragmentos de bosque que sostienen una economía maderera tradicional, transformando estos territorios en paisajes "aplanados" similares a los de la llanura pampeana . Estos factores dan como resultado que la degradación de ecosistemas, servicios ambientales, suelo y agua sean mucho más marcadas que en la pampa.

En segundo lugar, no sólo se desplazan especies, sino que hay un efecto de desplazamiento de población de pequeños campesinos y población indígena que vive en, y con, los recursos que provee el monte. Las transformaciones sociales derivadas de la agriculturización en regiones extra-pampeanas son similares: reestructuración social asociados a la concentración económica y productiva, activación del sector.

Con un escenario internacional con una alta demanda de alimentos, la situación es de una "gran oportunidad" para que la el país genere ingentes en divisas. El problema clave y quizás el más complejo de dilucidar es como van a usarse esos recursos. Si servirán para generar una ampliación de la brecha entre los sectores más

${ }^{15}$ Fernando Andrade et al., Los Desafíos de La Agricultura Argentina: Satisfacer Las Futuras Demandas y Reducir El Impacto Ambiental (Ciudad Autónoma de Buenos Aires: Ediciones INTA, 2017), 21. 
postergados y los más favorecidos o si se dispondrán para reducir las inmensas desigualdades socio-económicas engendradas en los últimas décadas.

Es importante señalar los riesgos que el proceso conlleva, ya que pueden afectar la sostenibilidad del mismo:

a) Tendencia al monocultivo de soja.

b) Reversión de las actuales tendencias climáticas

c) Migración por caída del trabajo rural y por la no inclusión de pequeños productores en el proceso.

d) La materia orgánica de los suelos y el balance de CO2.

e) Pérdida de biodiversidad

Este espacio complejo brindó amplias posibilidades de diversificación de la producción, tanto en relación a los tipos de recursos como a su forma de utilizarlos. Sin embargo, el modo de ocupación de la tierra y apropiación de los recursos ha generado la más notable de las asimetrías, como es el de una subregión hegemónica (la región pampeana), con monopolio de las ventajas económicas y sociales, en contraste con un interior supeditado a lo acaecido en el centro, del cual provienen en 90\% de las exportaciones. Este modelo de hegemonía de la región pampeana, produjo profundos desequilibrios regionales, originando un modelo de dependencia centroperiferia que ejerce una enorme influencia sobre los tipos de uso de la tierra y el manejo ambiental de las regiones extrapampeanas. Esto se manifiesta en el avance de la frontera agropecuaria hacia el Norte, como consecuencia de la agriculturización y de la sojización, con el desmonte de bosque natural y el traspaso acrítico de los paquetes tecnológicos pampeanos a las ecorregiones tropicales-subtropicales

¿Cuáles son entonces los principales impactos ambientales de esta transformación estructural de la agricultura argentina? 


\section{Consecuencias Socioambientales del Monocultivo de Soja}

\section{EL IMPACTO EN LOS SUELOS}

El monocultivo de soja causó desequilibrios agroecológicos sustanciales, como la pérdida de capacidad productiva de la tierra, aumentó la presión de plagas y enfermedades, cambios en la población de malezas, aumentó el riesgo de contaminación con pesticidas químicos, etc ${ }^{16}$.

En este proceso de agricultura industrial, el equilibrio de la materia orgánica tiende a ser perjudicial ya que el carbono mineralizado no se compensa con el aportado por el rastrojo de soja, mientras que una baja relación C / $\mathrm{N}$ caracteriza este cultivo. Si pensamos en la sostenibilidad agrícola a mediano y largo plazo, solo se puede garantizar con la diversificación de cultivos que en general preservan el medio ambiente y el suelo en particular, de una manera significativamente superior al caso de los monocultivos ${ }^{17}$. Este modelo agrícola promueve reducciones en la materia orgánica y diversas formas de deterioro estructural que favorecen el desarrollo de procesos erosivos en suelos expuestos ${ }^{18}$. De la superficie implantada en Argentina en las últimas dos décadas, el 30\% concierne a cereales y el 70\% a semillas oleaginosas, especialmente soja. Tres décadas atrás la relación era inversa. La dificultad está conectada con el hecho de que la soja tiene un sistema de raíces escasamente denso y devuelve una muy baja cantidad de desechos al suelo (rastrojo). Las aportaciones del rastrojo de la soja no solo son mínimas en cantidad, sino que también tienen una menor persistencia en el sistema que las de los otros cultivos, sobre todo por su significativo contenido de nitrógeno (baja relación $\mathrm{C} / \mathrm{N})^{19}$.

Asimismo, en este marco de creciente productividad, debemos entender que con la difusión del "agronegocio", los sistemas de producción sostenidos en las formas tradicionales, apoyados en la conformación de unidades campesinas entran en una zona de riesgo para su supervivencia, sumando además el hecho de que en estas

\footnotetext{
${ }^{16}$ A. Brown et al., La Situación Ambiental Argentina 2005 (Buenos Aires: Fundación Vida Silvestre Argentina, 2006).

17 Pengue, "Commoditización y Diversificación de La Producción Agropecuaria Frente a La Oferta Biotecnológica Argentina: Dos Alternativas Mutuamente Excluyentes?," 108.

${ }^{18}$ M. C. Sasal, A. Andriulo, and Miguel A. Taboada, "Soil Porosity Characteristics on Water Dynamics under Direct Drilling in Argiudolls of the Argentinean Rolling Pampas," Soil \& Tillage Research 87 (2006): 8-19.

19 G. Studdert and H. Echeverría, "Soja, Girasol y Maíz En Los Sistemas de Cultivos En El Sudeste Bonaerense," in Bases Para El Manejo Del Maíz, El Girasol y La Soja, ed. Fernando H. Andrade and Víctor O. Sadras, 2nd ed. (INTA-University of Mar del Plata, 2002).
} 
zonas la presencia de población de pueblos originarios es preponderante, las que a su vez mantienen relaciones extremadamente complejas y conflictivas con los mercados del modelo capitalista. Los éxodos, el despoblamiento rural, un paisaje desarticulado, son expresiones que difieren con el lucrativo paisaje de la soja, donde sobresalen grandes extensiones de tierra cubiertas de leguminosas o cereales dominantes, sostenidos por un equipamiento elementos tecnológico sofisticado, lo que marca el sostenido y profundo carácter transformador de ese modelo productivo, en relación a los aspectos socio-ambientales del lugar ${ }^{20}$.

\section{CONTAMINACIÓN: HERBICIDAS}

El rápido avance de la frontera agrícola ha convertido al "cultivo industrial" de la soja en el principal agente de destrucción del "Parque chaqueño"21. En la campaña de soya de 2017, se usó el equivalente a más de 300 millones de litros de glifosato, un herbicida que está relacionado con casos de cáncer, malformaciones, enfermedades alérgicas y otras, que afectan a los residentes, especialmente niños y mujeres, sujetos a las consecuencias de la fumigación en las proximidades o directamente de sus ciudades y pueblos. En 2017, se estimó que en el país, los se gastaron US\$ 2,381 millones en pesticidas, es decir, un equivalente a 317 millones de $\mathrm{kg} / 1$.

Estudios recientes en arroyos, ríos, aire y suelos de diferentes partes del país, en los que se evaluó el glifosato y su principal producto de degradación (AMPA, "ácido aminometilfosfónico") y otros agroquímicos, han indicado una alta frecuencia de presencia y concentraciones en el agua que generalmente exceden los umbrales establecidos como aceptables por países centrales ${ }^{22}$.

\footnotetext{
${ }^{20}$ Ana Isabel Rivas and Adriana del Valle Rodríguez, "El Cultivo de La Soja En El Norte Grande Argentino: Proceso de Crecimiento Espacial y Productivo," 2019, 10, http://www.observatoriogeograficoamericalatina.org.mx/egal12/Geografiasocioeconomica/Geografiaagricola/42.pdf.

${ }^{21}$ La región denominada Parque Chaqueño abarca aproximadamente 67.495 .995 ha. La parte argentina representa el $58 \%$ de la superficie del Gran Chaco Americano e involucra a la totalidad de las provincias de Formosa, Chaco y Santiago del Estero, al norte de las provincias de Santa Fe, San Luis y Córdoba, al este de las provincias de Salta, Tucumán, Catamarca, La Rioja y San Juan y noroeste de Corrientes.

22 Virginia C. Aparicio et al., "Environmental Fate of Glyphosate and Aminomethylphosphonic Acid in Surface Waters and Soil of Agricultural Basins," Chemosphere 93, no. 9 (November 2013): 1866-73, https://doi.org/10.1016/j.chemosphere.2013.06.041; Virginia C. Aparicio et al., "Glyphosate and AMPA Concentrations in Wind-Blown Material under Field Conditions," Land Degradation \& Development 29, no. 5 (May 2018): 1317-26, https://doi.org/10.1002//dr.2920; Carolina González et al., Relevamiento de La Utilizacion de Agroquimicos En La Provincia de Buenos Aires: Mapa de Situacion e Incidencia Sobre La Salud (Defensor del Pueblo Provincia de Buenos Aires / Univeersidad Nacional de la Plata, 2015).
} 
Gráfico 2. Evolución en el uso de agrotóxicos, número de litros / kg por año.

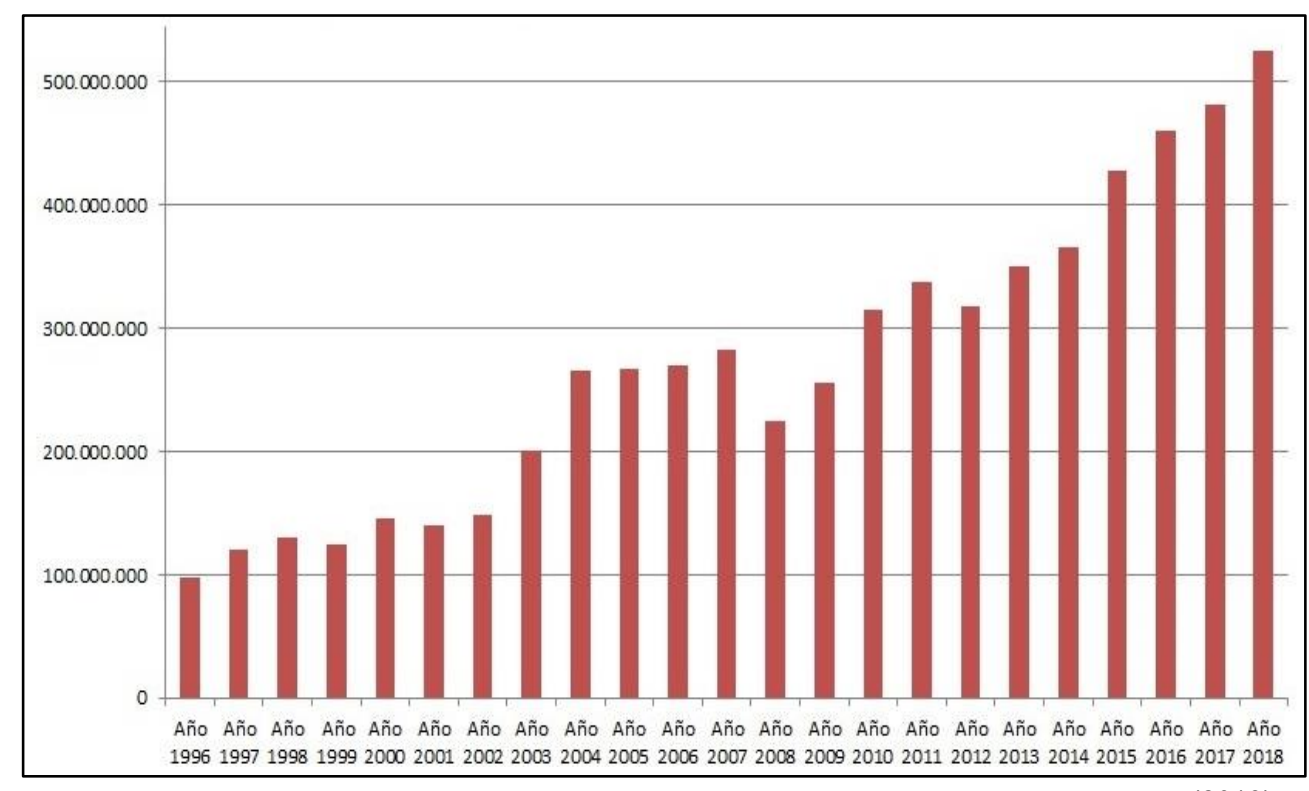

Fuente: Elaboración propia en base a datos de la Ministerio de Agroindustria. Buenos Aires (2018).

PÉRDIDA DE BIODIVERSIDAD Y SERVICIOS ECOSISTÉMICOS

El problema de la pérdida de biodiversidad y de los servicios ecosistémicos que derivó del progreso y crecimiento de la agricultura industrial en la Argentina se debió a al menos cuatro mecanismos críticos: la extensión o magnitud del proceso, su homogeneidad, la falta de criterios para proteger áreas críticas y la aplicación de un modelo basado excesivamente en tecnologías de entrada ${ }^{23}$. En este sentido, un enfoque de servicios ecosistémicos (ES) constituye un adelanto en la dicotomía producción-conservación, ya que habilita que las diversas funciones de los ecosistemas y espacios agrarios estén relacionadas con los beneficios que generan para los humanos ${ }^{24}$.

El avance de la frontera agrícola con la adopción de sistemas de producción extremadamente simplificados, un proceso asociado con la deforestación y el reemplazo de los ecosistemas originales, destruyó hábitats con el consecuente detrimento en los niveles de biodiversidad y generó emisiones de gases de efecto invernadero, excesos de agua y degradación del suelo. Este problema de caída de la

\footnotetext{
${ }^{23}$ Andrade et al., Los Desafíos de La Agricultura Argentina: Satisfacer Las Futuras Demandas y Reducir El Impacto Ambiental, 21.

24 Anthony McMichael et al., "Linking Ecosystem Services and Human Well-Being," in Ecosystems and Human Well-Being: Multi-Scale Assessments, ed. Doris Capistrano et al. (Washington DC: Island Press, 2005), 43-60; Andrade et al., Los Desafíos de La Agricultura Argentina: Satisfacer Las Futuras Demandas y Reducir El Impacto Ambiental, 21.
} 
diversidad biológica y de los servicios ecosistémicos que acompañó el proceso de difusión e aceleración de la agricultura industrial en Argentina, se debió a un conjunto de factores que actuaban a diversas escalas de espacio y tiempo. Argentina ocupa el noveno lugar en un área deforestada en los últimos 25 años. En ese período, más de 9 millones de hectáreas han sido deforestadas, lo que representa aproximadamente el $25 \%$ del área total actual de bosques nativos ${ }^{25}$.

Las ecorregiones argentinas más afectadas por el proceso de este modelo de "agricultura industrial" son la Pampeana en el centro del país, la Chaqueña y las Yungas, ambas del norte argentino, donde el impulso de nuevas alternativas productivas y económicas asociadas principalmente con la expansión del cultivo de soja con su "paquete de tecnología" determinó valores en la transformación y en el reemplazo de bosques y arbustales nativos sin precedentes ${ }^{26}$, así como el progreso de la agricultura en sistemas tradicionalmente ganaderos.

Una proporción significativa de las regiones de Chaqueña y Pampeana mostraron tendencias negativas en la provisión de SE reguladora entre 2000 y $2014^{27}$. El ES de una provisión de carne y granos en detrimento de los de regulación y cultura, la clave para las poblaciones locales ${ }^{28}$. Varios estudios demuestran el impacto de la deforestación en la biodiversidad ${ }^{29}$, la captura de carbono en la biomasa $^{30}$ y los suelos $^{31}$ y la salinización del suelo ${ }^{32}$ degradación y pérdida de fragmentos de pastizales, ecosistemas ribereños y de humedales y pérdidas de biodiversidad ${ }^{33}$. Estos procesos de degradación son indicativos de la disminución de la capacidad de los ecosistemas transformados para proporcionar ES. Esta afectación no se produce solo

\footnotetext{
${ }^{25}$ Andrade et al., Los Desafíos de La Agricultura Argentina: Satisfacer Las Futuras Demandas y Reducir El Impacto Ambiental.

${ }^{26}$ N. Ignacio Gasparri and H. Ricardo Grau, "Deforestation and Fragmentation of Chaco Dry Forest in NW Argentina (1972-2007)," Forest Ecology and Management 258, no. 6 (September 2009): 913-21, https://doi.org/10.1016/.foreco.2009.02.024; David Manuel-Navarrete and Gilberto Gallopín, Integración de Políticas, Sostenibilidad y Agriculturización En La Pampa Argentina y Áreas Extrapampeana (Santiago de Chile: NU. CEPAL, 2007).

27 José M. Paruelo et al., "An Integrative Index of Ecosystem Services Provision Based on Remotely Sensed Data," Ecological Indicators 71 (December 2016): 145-54, https://doi.org/10.1016/j.ecolind.2016.06.054.

${ }_{28}$ Matias E. Mastrangelo and Pedro Laterra, "From Biophysical to Social-Ecological Trade-Offs: Integrating Biodiversity Conservation and Agricultural Production in the Argentine Dry Chaco," Ecology and Society 20, no. 1 (2015): 1-8, https://doi.org/10.5751/ES-07186-200120.

29 lbid.

30 J.N. Volante et al., "Ecosystem Functional Changes Associated with Land Clearing in NW Argentina," Agriculture, Ecosystems \& Environment 154 (July 2012): 12-22, https://doi.org/10.1016/j.agee.2011.08.012.

31 Sebastián Horacio Villarino et al., "Deforestation Impacts on Soil Organic Carbon Stocks in the Semiarid Chaco Region, Argentina," Science of The Total Environment 575 (January 2017): 1056-65, https://doi.org/10.1016/j.scitotenv.2016.09.175.

32 M. L. Amdan et al., "Onset of Deep Drainage and Salt Mobilization Following Forest Clearing and Cultivation in the Chaco Plains (Argentina)," Water Resources Research 49, no. 10 (October 2013): 6601-12, https://doi.org/10.1002/wrcr.20516.

33 Mariano Codesido, Carlos González-Fischer, and David Bilenca, "Distributional Changes of Landbird Species in Agroecosystems of Central Argentina," The Condor 113, no. 2 (May 2011): 266-73, https://doi.org/10.1525/cond.2011.090190.
} 
en el ES regulatorio o cultural, sino también en la disposición (ES), o en otras palabras, en la capacidad del ecosistema para sostener la producción a largo plazo, especialmente cuando la agricultura avanzó en tierras marginales que afectan el ES de protección del suelo proporcionada por el ecosistema original.

Con esta expansión, los espacios donde persisten los sistemas productivos tradicionales sostenidos por las unidades campesinas y aquellos donde hay una población indígena que mantiene diferentes grados de articulación con las estructuras capitalistas se han puesto en riesgo.

La expansión del monocultivo de soja es el principal motor de degradación y disminución de ecosistemas autóctonos como bosques, humedales y praderas, lo que a su vez genera un daño de los bienes y servicios esenciales de los ecosistemas, para el bienestar de los seres humanos a la par que establece un modelo de desarrollo sostenible.

En el caso argentino, el impacto mas severo de la expansión de esta oleaginosa se ha dado en la región del "El Gran Chaco Americano". La misma es una de las regiones boscosas más extensa de Sudamérica junto a la Amazonía y al Cerrado. Abarca alrededor de 1 millón de $\mathrm{km}^{2}$, compartidos por Argentina (que comprende el 60\% de su extensión), Bolivia y Paraguay. Actualmente el 15\% de la región ha sido transformada para producción agrícola y ganadera, mostrando los índices más rápidos de conversión del mundo, con medio millón de hectáreas desmontadas entre 2010 y 2012 por la expansión de la agricultura mayormente impulsada por la soja. Una demanda creciente del mercado junto con innovaciones como las semillas genéticamente modificadas, la labranza cero y otros cambios tecnológicos han hecho que el cultivo sea más viable en aéreas más secas y tradicionalmente menos productivas como las provincias de la región semiárida del centro del país (Salta, Chaco, Santiago del Estero, Formosa y Tucumán) ${ }^{34}$.

Estas áreas de expansión de la frontera agropecuaria presentan un aumento conjunto del cultivo de soja y de la ganadería que va acompañado de una elevada tasa de deforestación. En Argentina, casi toda la soja es cultivada por grandes y medianos

${ }^{34}$ Adrián Zarrilli, "Ambiente, Producción y Mercado. El Impacto Transformador En Una Economía Periférica, El Gran Chaco Argentino En El Siglo XX," Áreas. Revista Internacional De Ciencias Sociales 35, no. 121-139 (2016), https://revistas.um.es/areas/article/view/279191. 
productores con un mínimo de 150 ha. El impacto social del sistema de producción de soja fue más brusco en la región semiárida del noroeste y centro del país, donde predominaban los pequeños productores con economías de subsistencia con un fuerte apoyo en la recolección de leña y uso del bosque para ganadería extensiva, los cuales se vieron afectados por la deforestación

Mientras cada vez más tierra está reservada a la producción de soja, ecosistemas representativos en Sudamérica están cada vez bajo una significativa presión del mercado. Durante las últimas décadas enormes superficies de bosque nativo y llanuras naturales han sido transformadas a la agricultura industrial, principalmente en países en vías de desarrollo. Esto ha ayudado a alimentar a la creciente población mundial y ha traído beneficios económicos a los países que la producen y la comercializan (por ejemplo Brasil, India o el propio caso de la Argentina). Pero la conversión de ecosistemas naturales lleva un costo alto. La biodiversidad declina, las poblaciones de especies en regiones tropicales han caído en promedio $60 \%$ desde 1970. La merma de bosques es un factor clave en el cambio climático, siendo responsable por el mas del $20 \%$ de las emisiones de gases con efecto invernadero. A la vez que los ecosistemas son devastados o degradados, dilapidamos muchos de los servicios de los cuales dependemos, desde agua limpia y suelos sanos hasta la polinización y el control de plagas. El desmonte de bosques y otros ecosistemas también tiene un impacto social: los bosques sudamericanos son el hogar de muchas comunidades indígenas, suministrándoles comida, albergue, combustible, medicinas y formas de ganarse la vida ${ }^{35}$.

La soja ha implicado además la expulsión y alejamiento de comunidades nativas en Argentina y Paraguay ${ }^{36}$. El boom de la este cultivo ha sido uno de los principales impulsores de la pérdida de ecosistemas naturales en Sudamérica en años recientes. El incremento inicial de la producción de soja en el continente coincidió, como ya señalamos con el desmonte de grandes áreas de ecosistemas nativos, para la agricultura. Asimismo, la escala del monocultivo de soja casi no ha tenido

\footnotetext{
35 FAO, "Servicios Ecosistémicos y Biodiversidad," Organización de las Naciones Unidas para la Alimentación y la Agricultura, n.d., http://www.fao.org/ecosystem-services-biodiversity/es/.

36 J. Hobbs, "Paraguay's Destructive Soy Boom," New York Times, 2012, http://www.nytimes.com/2012/ 07/03/opinion/paraguays-destructive-soyboom.html.
} 
antecedentes. Al igual que con cualquier sistema que cosecha un solo cultivo en áreas grandísimas, el monocultivo de soja minimiza los servicios ecológicos y se vuelve aceleradamente dependiente de los insumos químicos para controlar las plagas animales o vegetales. La escala del monocultivo mismo crea riesgos ecológicos, incluso nuevos, que solo "pueden" ser moderados con mayor intervención humana ${ }^{37}$.

El cambio hacia este modelo de agricultura industrial, generó además una inmensa transformación social, junto con muchos reclamos y controversias sobre los costos y los beneficios del patrón de desarrollo sojero. El mismo ha aumentado los niveles de la desigualdad social y afirmado el proceso de concentración de la tierra. La mayor porción de la producción de soja en Sudamérica, se realiza a una escala industrial, lo cual pone al pequeño productor en inferioridad, y genera la propagación de productores de mediana y gran escala que incitan a la concentración de la tierra, que, a su vez, desplaza a la población local y arrebata sus medios de vida ${ }^{38}$.

\section{CONCLUSIONES: ¿ES POSIBle LA "SOSTENIBILIDAD"?}

El cambio hacia este modelo de agricultura industrial generó un cambio social, junto con muchos reclamos y controversias sobre los costos y beneficios del modelo de desarrollo del cultivo de soja. Ha aumentado los niveles de desigualdad y ha consolidado el proceso de concentración de la tierra en pocas manos. La mayor parte de la producción de esta legumbre en América del Sur opera a escala industrial, lo que pone al pequeño productor en desventaja, y genera la expansión de productores de mediana y gran escala que estimulan la concentración de la tierra, lo que, a su vez, desplaza a la población local y arrebata sus medios de vida Cuando el sector productivo habla de sostenibilidad, se refiere principalmente a la sostenibilidad de la actividad productiva en sí misma, es decir, mantener o mejorar los rendimientos, conservar el suelo, utilizar adecuadamente los recursos hídricos, etc. Para este fin, se ha desarrollado una batería de buenas prácticas. desarrollado, incluyendo siembra directa y rotación de cultivos.

\footnotetext{
37 Walter A. Pengue, Agricultura Industrial y Transnacionalización En América Latina: ¿La Transgénesis de Un Continente? (México DF: UACMAPNUMA, 2005).

38 WWF, "El Crecimiento de La Soja: Impactos y Soluciones" (Gland, Suiza, 2014), https://www.wwf.org.ec/?217662/El-crecimiento-de-la-sojaimpactos-y-.
} 
Por otro lado, cuando el sector ambiental habla de sostenibilidad, habla principalmente del mantenimiento de los "bienes y servicios" de la naturaleza, es decir, la biodiversidad, la calidad del agua de los humedales, la liberación de carbono acumulado en los bosques y el respeto a los territorios rurales. comunidades En los entornos subtropicales, donde la soja se ha expandido en la última década, se concentran las áreas de mayor biodiversidad, humedales significativos y áreas de mayor concentración y diversidad indígena. Estas dos visiones de sostenibilidad son correctas y complementarias, y ambas son necesarias. La respuesta a esta aparente dicotomía está en la planificación territorial, llevada a cabo a escala de los territorios provinciales, que se lleva a la extensión de la tierra o "paisajes", generando espacios de planificación territorial que permiten asegurar que el desarrollo económico en sí mismo promueve las buenas prácticas agrícolas, el mantenimiento de las áreas de protección de la biodiversidad y la solución de conflictos territoriales con las poblaciones locales.

\section{REFERENCIAS}

Amdan, M. L., R. Aragón, E. G. Jobbágy, J. N. Volante, and J. M. Paruelo. “Onset of Deep Drainage and Salt Mobilization Following Forest Clearing and Cultivation in the Chaco Plains (Argentina)." Water Resources Research 49, no. 10 (October 2013): 6601-12. https://doi.org/10.1002/wrcr.20516.

Andrade, Fernando, Miguel Taboada, Daniel Lema, Nestor Maceira, Hernán Echeverría, Gabriela Posse, Daniel Prieto, et al. Los Desafíos de La Agricultura Argentina: Satisfacer Las Futuras Demandas y Reducir El Impacto Ambiental. Ciudad Autónoma de Buenos Aires: Ediciones INTA, 2017.

Aparicio, Virginia C., Silvia Aimar, Eduardo De Gerónimo, Mariano J. Mendez, and José L. Costa. "Glyphosate and AMPA Concentrations in Wind-Blown Material under Field Conditions." Land Degradation E Development 29, no. 5 (May 2018): 1317-26. https://doi.org/10.1002/ldr.2920.

Aparicio, Virginia C., Eduardo De Gerónimo, Damián Marino, Jezabel Primost, Pedro Carriquiriborde, and José L. Costa. "Environmental Fate of Glyphosate and Aminomethylphosphonic Acid in Surface Waters and Soil of Agricultural Basins." Chemosphere 93, no. 9 (November 2013): 1866-73. https://doi.org/10.1016/j.chemosphere.2013.06.041.

Branford, Sue. "Argentina's Bitter Harvest." NewScientist, 2004. https://www.newscientist.com/article/mg18224436-100-argentinas-bitter- 
harvest/.

Brown, A., U. Martinez Ortiz, M. Acerbi, and J. Corcuera. La Situación Ambiental Argentina 2005. Buenos Aires: Fundación Vida Silvestre Argentina, 2006.

Codesido, Mariano, Carlos González-Fischer, and David Bilenca. "Distributional Changes of Landbird Species in Agroecosystems of Central Argentina." The Condor 113, no. 2 (May 2011): 266-73. https://doi.org/10.1525/cond.2011.090190.

FAO. "Servicios Ecosistémicos y Biodiversidad." Organización de las Naciones Unidas para la Alimentación y la Agricultura, n.d. http://www.fao.org/ecosystemservices-biodiversity/es/.

Gasparri, N. Ignacio, and H. Ricardo Grau. "Deforestation and Fragmentation of Chaco Dry Forest in NW Argentina (1972-2007)." Forest Ecology and Management 258, no. 6 (September 2009): 913-21. https://doi.org/10.1016/j.foreco.2009.02.024.

Giraldo, Omar Felipe. Ecología Politica de La Agricultura. Agroecología y Posdesarrollo. San Cristóbal de Las Casas, Chiapas, México: El Colegio de la Frontera Sur, 2018.

González, Carolina, Patricio Alarcón, Gloria Castellano, Alejandro Echegaray, Rodolfo Iuliano, Ignacio Lara, Juan C. Colombo, et al. Relevamiento de La Utilizacion de Agroquimicos En La Provincia de Buenos Aires: Mapa de Situacion e Incidencia Sobre La Salud. Defensor del Pueblo Provincia de Buenos Aires / Univeersidad Nacional de la Plata, 2015.

Hobbs, J. “Paraguay's Destructive Soy Boom." New York Times, 2012. http://www.nytimes.com/2012/07/03/opinion/paraguays-destructive-soyboom.html.

Lema, Daniel. "Crecimiento y Productividad Total de Factores En La Agricultura: Argentina y Países Del Cono Sur 1961-2013." Argentina, 2015.

http://documents.worldbank.org/curated/pt/970151468197997810/Crecimiento -y-productividad-total-de-factores-en-la-agricultura-Argentina-y-países-delcono-sur-1961-2013.

Maarten Dros, Jan. Manejo Del Boom de La Soya: Dos Escenarios Sobre La Expansión de La Producción de La Soya En América Del Sur. Amsterdam: AIDEnvironment, 2004.

Manuel-Navarrete, David, and Gilberto Gallopín. Integración de Políticas, Sostenibilidad y Agriculturización En La Pampa Argentina y Áreas Extrapampeana. Santiago de Chile: NU. CEPAL, 2007.

Mastrangelo, Matias E., and Pedro Laterra. "From Biophysical to Social-Ecological Trade-Offs: Integrating Biodiversity Conservation and Agricultural Production in the Argentine Dry Chaco." Ecology and Society 20, no. 1 (2015): 1-8. https://doi.org/10.5751/ES-07186-200120.

McMichael, Anthony, Robert Scholes, Manal Hefny, Elvira Pereira, Cheryl Palm, and Simo Foale. "Linking Ecosystem Services and Human Well-Being." In Ecosystems 
and Human Well-Being: Multi-Scale Assessments, edited by Doris Capistrano, Cristián Samper K., Marcus J. Lee, and Ciara Raudsepp-Hearne, 43-60.

Washington DC: Island Press, 2005.

Merenson, Carlos. "Primera Estimación Del Pasivo Socio-Ambiental de La Expansióndel Monocultivo de Soja En Argentina." Ciencia \& Naturaleza 11 (2009): $1-7$.

Montenegro, Celina, Mabel Strada, Julieta Bono, N. Ignacio Gasparri, Eduardo Manghi, M. Gabriela Parmuchi, and Marcelo Brouver. "Estimación de La Pérdida de Superficie de Bosque Nativo y Tasa de Deforestación En El Norte de Argentina." Buenos Aires, 2005.

Morello, J. "Entrando Al Chaco Con y Sin El Consentimiento de La Naturaleza." Vida Silvestre 92 (2005): 23.

Nogués, Julio J. "Barreras Sobre Las Exportaciones Agropecuarias: Impactos Económicos y Sociales de Su Eliminación." Argentina, 2015.

http://documentos.bancomundial.org/curated/es/821761468000270036/Barre ras-sobre-las-exportaciones-agropecuarias-impactos-económicos-y-socialesde-su-eliminación.

Paruelo, José M., Marcos Texeira, Luciana Staiano, Matías Mastrángelo, Laura Amdan, and Federico Gallego. "An Integrative Index of Ecosystem Services Provision Based on Remotely Sensed Data." Ecological Indicators 71 (December 2016): 14554. https://doi.org/10.1016/j.ecolind.2016.06.054.

Pengue, Walter A. Agricultura Industrial y Transnacionalización En América Latina: ¿La Transgénesis de Un Continente? México DF: UACMAPNUMA, 2005.

---. "Commoditización y Diversificación de La Producción Agropecuaria Frente a La Oferta Biotecnológica Argentina: Dos Alternativas Mutuamente Excluyentes?" In Transgénicos. Biotecnología En El Agro, edited by Comisión de Agricultura de la Honorable Camara de Senadores de Buenos Aires, 107-24. Buenos Aires: Editorial Universitaria de La Plata, 2000.

Piñeiro, Martín, and Feredico Villarreal. "Modernización Agrícola y Nuevos Actores Sociales." Revista Ciencia Hoy En Línea 15, no. 87 (2005): 32-36.

Rabinovich, Jorge E., and Filemón Torres. "Caracterización de Los Síndromes de Sostenibilidad Del Desarrollo. El Caso de Argentina, Santiago, ECLAC, the Case of Buenos Aires." Environment and Urbanization 12, no. 2 (2004).

Rivas, Ana Isabel, and Adriana del Valle Rodríguez. "El Cultivo de La Soja En El Norte Grande Argentino: Proceso de Crecimiento Espacial y Productivo," 2019. http://www.observatoriogeograficoamericalatina.org.mx/egal12/Geografiasocio economica/Geografiaagricola/42.pdf.

Sasal, M. C., A. Andriulo, and Miguel A. Taboada. "Soil Porosity Characteristics on Water Dynamics under Direct Drilling in Argiudolls of the Argentinean Rolling 
Pampas." Soil \& Tillage Research 87 (2006): 8-19.

Shiva, Vandana. Los Monocultivos de La Mente. Monterrey, Mexico: Editorial Fineo, 2007.

Studdert, G., and H. Echeverría. "Soja, Girasol y Maíz En Los Sistemas de Cultivos En El Sudeste Bonaerense." In Bases Para El Manejo Del Maíz, El Girasol y La Soja, edited by Fernando H. Andrade and Víctor O. Sadras, 2nd ed. INTA-University of Mar del Plata, 2002.

Villarino, Sebastián Horacio, Guillermo Alberto Studdert, Pablo Baldassini, María Gabriela Cendoya, Lucía Ciuffoli, Matias Mastrángelo, and Gervasio Piñeiro. "Deforestation Impacts on Soil Organic Carbon Stocks in the Semiarid Chaco Region, Argentina." Science of The Total Environment 575 (January 2017): 1056-65. https://doi.org/10.1016/j.scitotenv.2016.09.175.

Volante, J.N., D. Alcaraz-Segura, M.J. Mosciaro, E.F. Viglizzo, and J.M. Paruelo. "Ecosystem Functional Changes Associated with Land Clearing in NW Argentina." Agriculture, Ecosystems \& Environment 154 (July 2012): 12-22. https://doi.org/10.1016/j.agee.2011.08.012.

WWF. "El Crecimiento de La Soja: Impactos y Soluciones." Gland, Suiza, 2014. https://www.wwf.org.ec/?217662/El-crecimiento-de-la-soja-impactos-y-.

Zarrilli, Adrián. "Ambiente, Producción y Mercado. El Impacto Transformador En Una Economía Periférica, El Gran Chaco Argentino En El Siglo XX." Áreas. Revista Internacional De Ciencias Sociales 35, no. 121-139 (2016). https://revistas.um.es/areas/article/view/279191. 


\title{
Nature put in Check: The Expansion of the Agricultural Frontier in Argentina and its Socio-Environmental Impact (1980-2017)
}

\begin{abstract}
RESUMEN
The expansion of the agricultural frontier associated with the notable growth of agricultural products in Argentina at the end of the XX and XXI centuries, driven mainly by the significant expansion of soybean cultivation, has produced one of the most substantial economic, social, demographic and environmental in contemporary Argentina. Soy, as the dominant crop of the last decades in Argentine agriculture, has been weighted by some sectors and demonized by others. For some, it is the crop that has allowed the recovery of the Argentine economy due to its high productivity, profitability and its positive impact on the trade balance of the last two decades. They emphasize the flexibility of the crop, the simplicity of its management and the high incidence of its technological package, sustained in a model integrated by direct sowing, transgenic seeds and the generalized application of herbicides. For others, on the other hand, the cultivation of this oilseed triggers the rapid deforestation of native forests, concentrates wealth in large corporations, destroys rural jobs, expels indigenous communities, annihilates biological diversity, deteriorates the environmental quality and affects human health. How much myth and reality is there in this debate often skewed by economic, political or ideological interests? This work seeks to clarify some critical and controversial aspects of this debate. It is vital to try to answer some substantial questions: How much of apology and reality are there in the environmental impacts of this soy model, concerning biodiversity, the use of pesticides and changes in habitat? What social transitions does it produce in the rural area of Argentina? How do ecological and environmental indicators compare with other countries of the subcontinent and the world? None of these problems has a simple answer, but it is possible to try to shape it by analyzing 30 years of progress on the agricultural frontier.
\end{abstract}

Keywords: Argentina; Chaquean Región; Soy, Environmental. 